\title{
配体促进钴催化的烯烃自由基型氢胺化反应
}

\author{
刘冰雪刘 强*
}

(清华大学化学系 基础分子科学中心 北京 100084)

\section{Ligand-Promoted Cobalt-Catalyzed Radical Hydroamination of Alkenes}

\author{
Liu, Bingxue Liu, Qiang* \\ (Center of Basic Molecular Science, Department of Chemistry, Tsinghua University, Beijing 100084)
}

胺及其衍生物普遍存在于药物、天然产物和功能材 料分子中，因此从简单易得的原料出发高效合成胺类化 合物长期以来都是有机化学领域的重要研究方向. 其 中, 烯烃的氢胺化反应是合成含氮分子最为直接和有效 的方法之一 ${ }^{[1 \sim 3]}$, 一直受到研究者的广泛关注. 在传统 的烯烃活化策略中, 烯烃迁移插入 $\mathrm{M}-\mathrm{H}$ 形成烷基金属 中间体是反应选择性控制的关键步骤, 存在难以控制化 学、区域、立体选择性的多重挑战(Scheme 1a), 特别是 无鳌合基团参与、非活化烯烃的高选择性分子间氢胺化 反应依然是难以实现的.

(a) Transition-metal-catalyzed hydroamination of alkenes

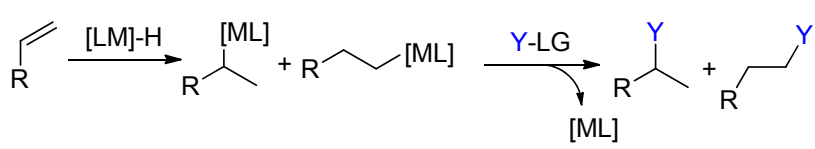

(b) Metal-catalyzed alkene radical hydroamination via HAT

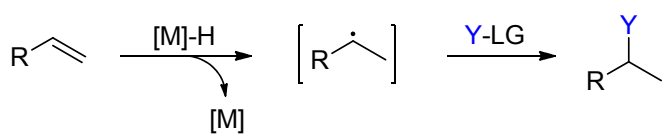

(c) Ligand-promoted metal-catalyzed alkene radical hydroamination via HAT

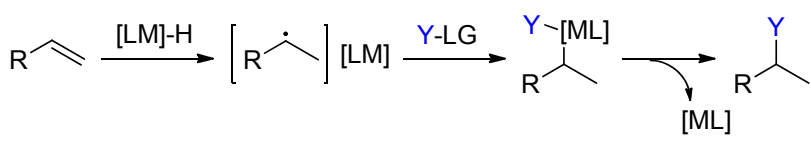

图式 1 金属催化的烯烃氢胺化反应策略

Scheme 1 Strategies for metal-catalyzed alkenes hydroamination

金属催化的氢原子转移反应(HAT)通过稳定自由基 中间体的生成, 表现出优异的 Markovnikov 区域选择性
和化学选择性 ${ }^{[4,5]}$ (Scheme 1b), 然而, 由于反应生成高 活性的自由基中间体，导致反应过程立体选择性控制更 加困难，同时，缺乏合适的手性配体使得实现不对称的 烯烃自由基型氢胺化反应具有很大的挑战性. 因此, 发 展一种高效的手性配体是解决上述问题的关键 (Scheme 1c).

浙江大学化学系陆展课题组之前成功发展了一系 列非对称三齿含氮(UNT)手性配体，实现了非活化烯烃 的不对称氢化 ${ }^{[6]}$ 、硼氢化 ${ }^{[7]}$ 、硅氢化 ${ }^{[8]}$ 等氢官能团化反应. 在此基础上，他们进一步发展了UNT 型配体，该类配体 可以很好地促进钴催化的烯烃自由基式氢胺化反应. 该 反应表现出优异的底物实用性, 实现了非活化末端烯烃 的高选择性分子间氢胺化反应(Scheme 2). 随后他们进 一步发展了手性 UNT 配体, 首次实现了简单末端烯烃 的自由基式不对称氢胺化反应 ${ }^{[9]}$, 最高 $e e$ 值可达 $85 \%$. 由于多数产物为固体, 所以可以通过一步重结晶过程方 便地将产物的对映体纯度提高到 $95 \%$ 以上. 值得一提的 是，该方法可以成功地应用于大宗化工原料 1-丁烯的不 对称氢胺化反应，有效地制备重要合成中间体手性 2-丁 胺.

此外，作者也开展了相关的合成应用研究，成功将 该方法用于氯苄雷司、 $(+/-)$ - 芬地林、 $(+/-)-N P S$ 、 $(S, R)$ 或 $(R, R)$ - 拉贝洛尔 ${ }^{[10]}$ 等药物分子的高效合成 (Scheme 3). 通过自由基捕获和自由基钟等机理研究实 验, 表明反应经历了一个氢原子转移(HAT)形成烷基自 由基中间体的过程. 具体可能的反应机理如 Scheme 4 所示.

综上所述，陆展课题组发展了一类高效的 UNT 型

* Corresponding author. E-mail: qiang_liu@mail.tsinghua.edu.cn. Published online February 26, 2020. 


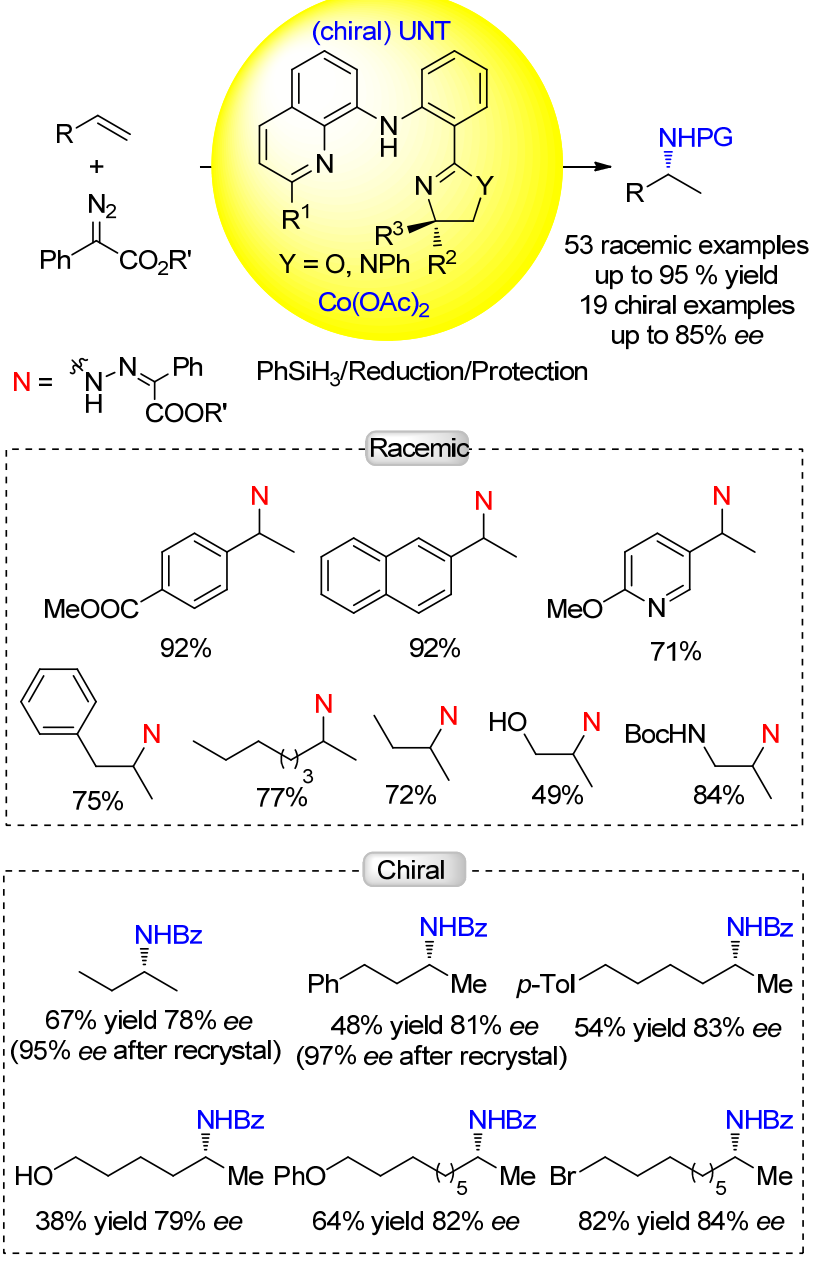

图式 2 钴催化的烯烃氢胺化反应

Scheme 2 Cobalt-catalyzed hydroamination of alkenes<smiles>COc1cccc(C(NCCCc2ccccc2Cl)c2ccccc2)c1</smiles>



$97 \%$ ee after recystal

$(S, R)$ or $(R, R)$-labetalol

图式 3 药物分子的合成

Scheme 3 Synthesis of drug molecules

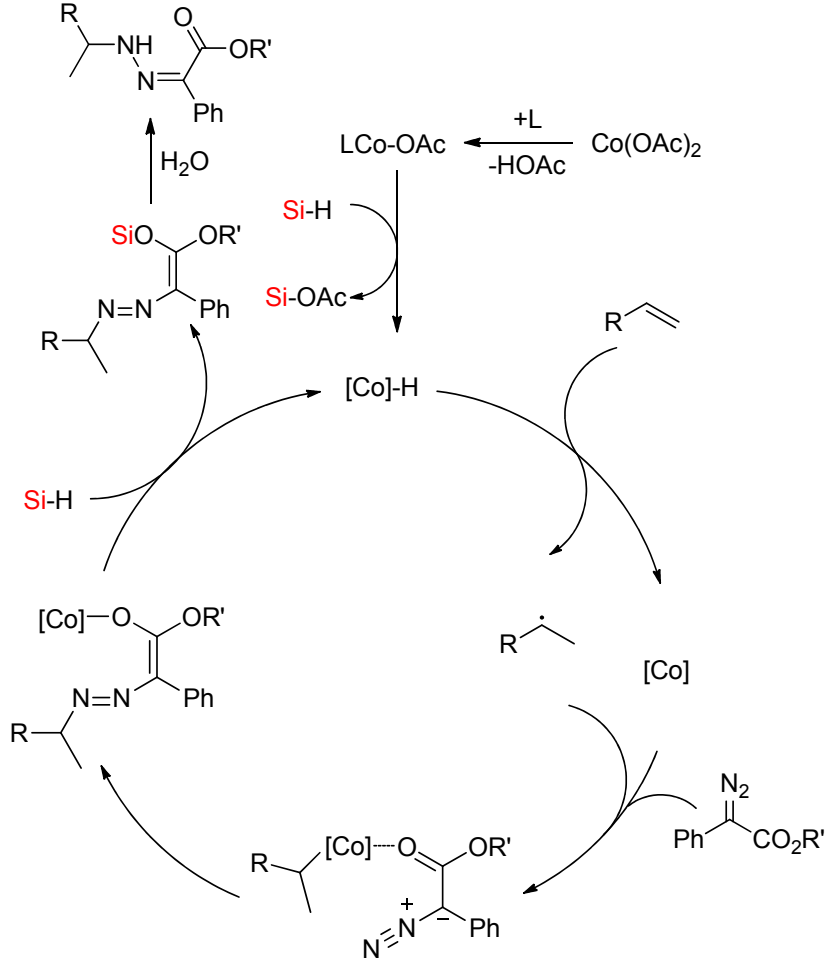

图式 4 可能的机理

Scheme 4 Proposed mechanism

配体，首次实现了非活化末端烯烃的自由基型不对称分 子间氢胺化反应. 该反应官能团兼容性强, 并具有优异 的 Markovnikov 区域选择性和立体选择性. 因此, 本工 作为解决了烯烃氢胺化反应领域的难题提供了一种新 策略, 极大地促进了非活化烯烃氢胺化反应的发展.

\section{References}

[1] Miki, Y.; Hirano, K.; Satoh, T.; Miura, M. Angew. Chem., Int. Ed. 2013, 52, 10830 .

[2] Zhu, S.; Niljianskul, N.; Buchwald, S. L. J. Am. Chem. Soc. 2013, $135,15746$.

[3] Yang, Y.; Shi, S.-L.; Niu, D.; Liu, P.; Buchwald, S. L. Science 2015, 349,62 .

[4] Gui, J.; Pan, C.-M.; Jin, Y.; Qin, T.; Lo, J. C.; Lee, B. J.; Spergel, S. H.; Mertzman, M. E.; Pitts, W. J.; La Cruz, T. E.; Schmidt, M. A.; Darvatkar, N.; Natarajan, S. R.; Baran, P. S. Science 2015, 348, 886.

[5] Crossley, S. W. M.; Obradors, C.; Martinez, R. M.; Shenvi, R. A. Chem. Rev. 2016, 116, 8912.

[6] Chen, J.; Chen, C.; Ji, C.; Lu, Z. Org. Lett. 2016, 18, 1594.

[7] Chen, X.; Cheng, Z.; Guo, J.; Lu, Z. Nat. Commun. 2018, 9, 3939.

[8] Cheng, B.; Liu, W.; Lu, Z. J. Am. Chem. Soc. 2018, 140, 5014.

[9] Shen, X.; Chen, X.; Chen, J.; Sun, Y.; Cheng, Z.; Lu, Z. Nat. Commun. 2020, 11, 783 .

[10] O'Reilly, M. E.; Dutta, S.; Veige, A. S. Chem. Rev. 2016, 116, 8105.

(Cheng, F.) 